Fetal Diagnosis and Therapy
Fetal Diagn Ther

DOI: $10.1159 / 000441027$
Received: May 27, 2015

Accepted after revision: September 7, 2015 Published online: October 1, 2015

\title{
First-Trimester Placental Growth Factor in Screening for Gestational Diabetes
}

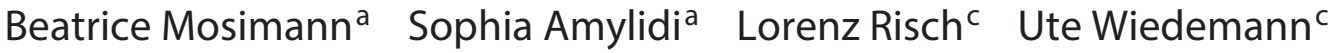 \\ Daniel Surbek $^{\mathrm{a}}$ Marc Baumann $^{\mathrm{a}}$ Christoph Stettler $^{\mathrm{b}}$ Luigi Raio $^{\mathrm{a}}$ \\ Departments of a Obstetrics and Gynecology and ${ }^{b}$ Endocrinology, University Hospital and University of Bern, and \\ 'Division of Clinical Chemistry, Labormedizinisches Zentrum Dr. Risch, Bern, Switzerland
}

\section{Key Words}

Placental growth factor · First trimester - Maternal serum screening $\cdot$ Gestational diabetes $\cdot$ Glycosylated hemoglobin

\begin{abstract}
Objective: The aim of this study was first to assess whether first-trimester serum concentrations of placental growth factor (PIGF) differ between patients with and without gestational diabetes (GDM) and second to test whether there is a correlation between glycosylated hemoglobin ( $\mathrm{HbA1c}$ ), a factor recently shown to be useful in predicting GDM, and PIGF. Methods: PIGF was measured at 8-14 weeks with the Kryptor Immunoassay Analyzer (Brahms, Berlin, Germany). Absolute values were converted to multiples of the median using the software provided by the Fetal Medicine Foundation London. GDM was diagnosed using internationally accepted criteria. $\mathrm{HbA} 1 \mathrm{c}$ levels were quantified using the TOSOH G7 automated hemoglobin analyzer. Results: From January to December 2014, 328 women were included in the study, 51 (15.5\%) of whom developed GDM. First-trimester PIGF quantification does not discriminate between women at risk to develop GDM and controls, while $\mathrm{HbA} 1 \mathrm{c}$ is able to do so. No correlation was found between PIGF and HbA1c. Conclusion: Our findings do not lend support to the hypothesis that early PIGF values are different in women who later develop GDM.

(c) 2015 S. Karger AG, Basel
\end{abstract}

\section{Introduction}

Overweight, obesity and associated metabolic disorders such as diabetes and cardiovascular disorders are increasing worldwide in a pandemic manner and affect also the fertile population, increasing the prevalence of complications such as gestational diabetes (GDM) and hypertensive disorders of pregnancy in particular [1]. GDM is defined as glucose intolerance with first onset or recognition during pregnancy. As glycemic changes become overt in the second half of pregnancy, the International Association of the Diabetes and Pregnancy Study Groups (IADPSG) has recommended a general screening for GDM using a 75-g oral glucose tolerance test (oGTT) at 24-28 weeks of gestation [2], based on the findings of the Hyperglycemia and Adverse Pregnancy Outcome (HAPO) study [3]. However this metabolic disorder may already be present preconceptionally as impaired fasting glucose, impaired glucose tolerance or unrecognized preexisting diabetes mellitus type 2 [4]. Therefore, international and national societies have recommended screening for these pre-existing metabolic disorders at the first antenatal visit by a fasting glucose measurement, $75-\mathrm{g}$ oGTT, or by assessment of glycosylated hemoglobin (HbA1c) $[2,5,6]$. Recently an association been first-trimester $\mathrm{HbA} 1 \mathrm{c}$ and GDM was reported [7, 8].

\section{KARGER 125}

(c) 2015 S. Karger AG, Base

$1015-3837 / 15 / 0000-0000 \$ 39.50 / 0$ 
Placental growth factor (PlGF) belongs to the vascular endothelial growth factor (VEGF) family and is an angiogenic factor that stimulates endothelial cells via fms-like tyrosine kinase-1. PlGF is expressed in trophoblastic cells and is suggested to play a vital role in the development of the placental vasculature [9]. Hypoxia and inflammation alter angiogenic and anti-angiogenic factors in the placenta [10], and maternal serum levels of PlGF are reduced in the first trimester of pregnancies that will develop preeclampsia (PE) or small for gestational age infants $[11,12]$. While earlier studies reported increased PlGF levels in pre-gestational diabetes (PGDM) and GDM [13], a recent publication demonstrated that in PGDM first-trimester maternal serum levels of PlGF are reduced [14], while yet another study showed again increased PlGF levels in pregnancies that developed GDM [15].

As there are still only limited and somehow also conflicting data available about first-trimester PlGF in GDM, the purpose of this study was to add data by comparing first-trimester PlGF in the general low-risk setting of our outpatient women who later developed GDM to those who did not, and to test whether a correlation exists between $\mathrm{HbAlc}$ and PlGF in the first trimester, as HbAlc is known to be increased in women who later develop GDM.

\section{Subjects and Methods}

In this prospective study we included consecutive pregnant women attending for their first routine antenatal visit at 8-14 weeks gestation who accepted to have a blood test for PlGF and HbAlc and a 75-g oGTT at 24-28 weeks gestation to diagnose GDM. As part of routine PE screening most women had a second PlGF measured at 11-14 weeks if the first one had been taken before 11 weeks gestation, as there are too limited existing data on the performance of PlGF drawn before 11 weeks for PE screening. Patients were recruited between January and December 2014 at the outpatient clinics of the Department of Obstetrics and Gynecology of the University Hospital Bern. Written informed consent was obtained from the women agreeing to participate in the study, which was approved by the Ethics Committee of the Canton of Bern. Exclusion criteria were pre-existing diabetes type 1 or 2 and also women with a first-trimester $\mathrm{HbAlc}$ value of $\geq 6.5 \%$.

Maternal serum samples for PlGF were assayed with the Kryptor Immunoassay Analyzer (Brahms, Berlin, Germany) and results were expressed as absolute values and as multiples of the median (MoM) at 11-14 weeks only using The Fetal Medicine Foundation software (www.fetalmedicine.org). The lower detection limit was $3.6 \mathrm{pg} / \mathrm{ml}$.

$\mathrm{HbA} 1 \mathrm{c}$ levels were quantified using the TOSOH G7 automated hemoglobin analyzer. The method principle is based on high-performance liquid chromatography and is fully traceable IFCC standard in accordance with the European Union directive 98/79/EC on in vitro diagnostic medical devices. The $\mathrm{HbA} 1 \mathrm{c}$ level is given in percent and SI units ( $\mathrm{mmol} / \mathrm{mol})$ [16].

According to current guidelines $[2,3]$ the diagnosis of GDM was made when any of the following criteria were met on the $75-\mathrm{g}$ oGTT: fasting plasma glucose $\geq 5.1 \mathrm{mmol} / 1$, or glucose $\geq 10 \mathrm{mmol} / \mathrm{l}$ at $1 \mathrm{~h}$, or glucose $\geq 8.5 \mathrm{mmol} / \mathrm{l} 2 \mathrm{~h}$. Blood was also drawn from the antecubital vein. Plasma glucose was estimated using the Roche Modular Analytics Chemistry Systems. The principle of the method is enzymatic utilizing hexokinase, which catalyzes the phosphorylation of glucose to glucose-6-phosphate [17].

Statistical analyses were performed with GraphPad Prism version 5.0 for Windows (GraphPad Software, San Diego, Calif., USA). Correlations were explored using the nonparametric Spearman rank test, while proportions were analyzed using Fisher exact or $\chi^{2}$ test where appropriate. Mann-Whitney $U$ test was used to compare continuous variables. Statistical significance was considered achieved when $\mathrm{p}$ was $<0.05$.

\section{Results}

From January to December 2014 we included 328 women in our study. Of those 51 (15.5\%) fulfilled the criteria for GDM and 277 served as controls. The demographic characteristics of the study population are depicted in table 1. As expected, women with GDM were older and had a significantly higher body mass index (BMI) than those in the control group.

310 women had PlGF assessed at 11-14 weeks (50 with GDM), 131 had an additional PlGF measurement at $8+0$ to $10+6$ weeks gestation and 18 women had only a PlGF at $8-11$ weeks, adding up to 149 patients with a PlGF measurement before 11 weeks gestation ( 28 with GDM).

$\mathrm{HbA1c}$, always measured at the same time as the first drawn PlGF between $8+0$ and 14 weeks gestation, was analyzed in 262 out of the 328 women.

In table 2 the results of first-trimester $\mathrm{HbA} 1 \mathrm{c}$ and PlGF and PlGF MoM, dichotomized between women who did and those who did not develop GDM, are presented. PlGF was converted to MoM at 11-14 weeks only as there is too limited information on PlGF MoM before that gestational age. There was no difference in gestational age or crown-rump length between the groups. No difference was found comparing first-trimester PlGF between the groups (fig. 1), while HbAlc significantly discriminated between women who developed GDM and those who did not. There is no significant correlation between the absolute values of PlGF and HbAlc $(r=0.09, p=0.145)$ or between PlGF MoM and HbAlc $(r=0.08, p=0.172)$. Similarly, no correlation was found between $\mathrm{HbAlc}$ and PlGF in the GDM group only $(r=0.20, p=0.20)$ or in the control group only $(r=0.09, p=0.55)$. 
Table 1. Maternal demographic characteristics in comparison between the GDM and the control group

\begin{tabular}{lccc}
\hline & GDM $(\mathrm{n}=51)$ & Control $(\mathrm{n}=277)$ & p value \\
\hline Maternal age, years & $32.22 \pm 4.81$ & $30.65 \pm 5.52$ & $0.034^{*}$ \\
\hline Maternal BMI & $27.02 \pm 6.32$ & $23.1 \pm 4.34$ & $<0.0001^{*}$ \\
\hline Nulliparity & $17(33.3 \%)$ & $145(52.3 \%)$ & $0.015^{*}$ \\
\hline Maternal ethnicity & & & 0.409 \\
$\quad$ White & $33(64.7 \%)$ & $196(70.6 \%)$ & 0.680 \\
$\quad$ Black & $9(17.6 \%)$ & $43(15.5 \%)$ & 0.114 \\
$\quad$ South Asian & $2(11.8 \%)$ & $15(5.4 \%)$ & 0.750 \\
$\quad$ East Asian & $1(2.0 \%)$ & $5(1.8 \%)$ & 1.000 \\
$\quad$ Mixed & 2 & 28 & 0.195 \\
\hline Smoking & 1 & 4 & 0.573 \\
\hline Chronic hypertension & 4 & 20 & 0.776 \\
\hline Conception by ART & $38.56 \pm 1.90$ & $39.54 \pm 1.52$ & $0.0007^{*}$ \\
\hline Outcomes & $3.130 \pm 0.57$ & $3.290 \pm 0.50$ & 0.194 \\
$\quad$ GA at delivery, weeks gestation & $6.9 \%$ & $4.5 \%$ & 0.634 \\
Birthweight, kg & & $5.5 \%$ & \\
\hline
\end{tabular}

Comparisons between each outcome group with controls: all values are given as absolutes and percent or mean \pm SD. $\chi^{2}$ test and Fisher exact test for categorical variables and Mann-Whitney U test.

$\mathrm{ART}=$ Assisted reproductive technology; $\mathrm{GA}=$ gestational age; LGA $=$ large for gestational age. ${ }^{*} \mathrm{p}<0.05$.

Table 2. Distributions of biochemical parameters in the GDM and in the control group

\begin{tabular}{lccc}
\hline & GDM $(\mathrm{n}=51)$ & Control $(\mathrm{n}=277)$ & p value \\
\hline Crown-rump length at $11-14$ weeks, mm & $64.49 \pm 8.33$ & $65.17 \pm 8.23$ & 0.602 \\
\hline GA at study inclusion, weeks gestation & $10.8 \pm 1.46$ & $11.21 \pm 1.53$ & 0.094 \\
\hline $\begin{array}{l}\text { PlGF, pg/ml } \\
\quad \text { At } 8+0 \text { to } 14 \text { weeks }\end{array}$ & $34.30 \pm 18.65$ & $36.10 \pm 24.47$ & 0.818 \\
$\quad$ At 11 to 14 weeks & $44.13 \pm 21.23$ & $44.95 \pm 24.75$ & 0.729 \\
$\quad$ At $8+0$ to $10+6$ weeks & $23.44 \pm 8.41$ & $24.13 \pm 9.90$ & 0.926 \\
\hline PlGF MoM at 11 to 14 weeks & $1.05 \pm 0.38$ & $1.03 \pm 0.48$ & 0.454 \\
\hline HbA1c, \% & $5.39 \pm 0.37$ & $5.18 \pm 0.28$ & $0.0007^{*}$ \\
\hline
\end{tabular}

Comparisons between each outcome group with controls by Mann-Whitney U test: all values are given as mean \pm SD.

$\mathrm{GA}=$ Gestational age. ${ }^{*} \mathrm{p}<0.05$.

\section{Discussion}

The incidence of GDM in our collective is in accordance with the described incidence of $17.5 \%$ (9.3-25.5\%) in the IADPSG collaborating centers [18]. Our findings do not show any difference of first-trimester PlGF concentrations or their MoM between pregnant women who later developed GDM and controls, contradicting a recently published study that presented significantly higher first-trimester PlGF values in the GDM group [15]. The 


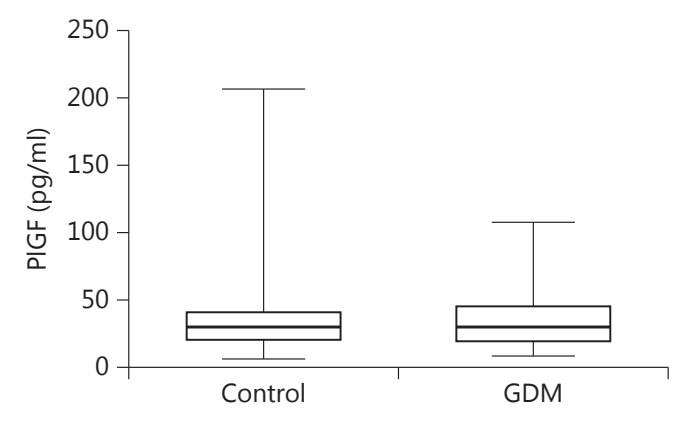

Fig. 1. Comparison of first-trimester PlGF concentrations in women with later GDM and in controls.

diagnosis of GDM in that study was also based on the HAPO criteria. However, no information was provided on how cases and controls were recruited.

Ong et al. [13] examined serum PlGF at 11-14 weeks gestation in 82 women with diabetic pregnancies consisting of 32 women with PGDM and 50 high-risk women with GDM diagnosed with a 75-g oGTT and by applying the World Health Organization criteria. They also found increased serum PlGF in PGDM, at least in the non-insulin-dependent subgroup. Newer data however found that serum PlGF was decreased in women with diabetes mellitus type 1 and in those with type 2 disease requiring treatment with insulin [14].

Eleftheriades et al. [15] explained their finding of higher first-trimester serum PlGF in pregnancies that later developed GDM by a hyperglycemia-induced alteration in placental angiogenesis. They based this assumption on a correlation they found between fasting oGTT glucose values and first-trimester PlGF concentration. It is well established that hyperglycemia affects angiogenesis [19]. In diabetic retinopathy VEGF is upregulated, while decreased VEGF levels contribute to impaired wound healing in diabetes [19]. Vasculogenesis and angiogenesis are essential for placental development and the VEGF family has been shown to play a key role [20]. Moreover, a recent metaanalysis of studies investigating postpartum placental histology derived from pregnancies complicated by PGDM or GDM demonstrates that there is increased placental volume, higher incidence of villous immaturity and increased angiogenesis [21]. Particularly the fetoplacental vasculature and endothelium were shown to be susceptible to hyperglycemia [22]. Fetal hyperinsulinism as a result of ma- ternal hyperglycemia stimulates placental angiogenesis [23]. Therefore, an increase in angiogenic markers such as PlGF might be a consequence of these alterations. However, PlGF is not altered in umbilical cord blood serum of neonates born to diabetic mothers [24]. Moreover, Tsiakkas et al. [14] found that first-trimester maternal PlGF serum concentration was decreased in pregnancies complicated by diabetes mellitus type 1 , and also in type 2 when treatment with insulin was required. Using three-dimensional sonography and power Doppler, it could be shown that first-trimester placental vascularization in pregnancies with PGDM is reduced, explaining in part the increased incidence of pregnancy-associated hypertensive disorders in diabetic pregnancies [25] and the lower first-trimester maternal serum PlGF found in both PE [26] and PGDM [14]. However, these findings also remain contradictory, as Cohen et al. [27], examining women with PGDM, could not demonstrate a difference in PlGF in early pregnancy in women who developed PE compared to women who remained normotensive or even compared to pregnant controls without diabetes, while later in pregnancy there was a significant change among the different groups.

A possible explanation for these divergent results in the literature and our findings might be that we excluded most of the possible cases with PGDM by screening for them using first-trimester $\mathrm{HbAlc}$ and excluding women with an $\mathrm{HbA} 1 \mathrm{c}$ of $\geq 6.5 \%$ according to the recommendation of the American Diabetes Association [5] and the International Expert Committee on Diabetes [28]. However, even after excluding PGDM, there remain some first-trimester metabolic changes in pregnancies that will develop GDM, as HbA1c was elevated in our and other studies $[7,8]$. The fact that we found no correlation between $\mathrm{HbAlc}$ and PlGF in the first trimester further lends support to the conclusion that PlGF in the first trimester is not a good marker for GDM.

In the time of turning the pyramid of obstetric care [29], early screening for GDM with timely intervention is desirable. Besides HbA1c, several first-trimester markers for GDM such as adiponectin, SHBG, TNRF1 and PAI2 have been described [30]. While first-trimester maternal serum PlGF is an excellent marker for PE [26], our results could not confirm any use of first-trimester PlGF in screening for GDM.

In conclusion, contradictory to previously published data, our results do not lend support to the theory that maternal serum PlGF is altered in the first trimester in pregnancies that will develop GDM. We also could not find any correlation between first-trimester $\mathrm{HbAlc}$, in our study an early marker for GDM, and PlGF. 


\section{Acknowledgement}

The Laboratory Medical Center Dr. Risch supported this study.

\section{Disclosure Statement}

Analysis of PlGF was performed by L. Risch's laboratory.

\section{References}

1 Barbour LA: Changing perspectives in preexisting diabetes and obesity in pregnancy: maternal and infant short- and long-term outcomes. Curr Opin Endocrinol Diabetes Obes 2014;21:257-263.

2 International Association of Diabetes and Pregnancy Study Groups Consensus Panel, Metzger BE, Gabbe SG, Persson B, Buchanan TA, Catalano PA, Damm P, Dyer AR, Leiva AD, Hod M, Kitzmiler JL, Lowe LP, McIntyre HD, Oats JJ, Omori Y, Schmidt MI: International Association of Diabetes and Pregnancy Study Groups recommendations on the diagnosis and classification of hyperglycemia in pregnancy. Diabetes Care 2010;33:676-682.

3 HAPO Study Cooperative Research Group, Metzger BE, Lowe LP, Dyer AR, Trimble ER, Chaovarindr U, Coustan DR, Hadden DR, McCance DR, Hod M, McIntyre HD, Oats JJ, Persson B, Rogers MS, Sacks DA: Hyperglycemia and adverse pregnancy outcomes. N Engl J Med 2008;358:1991-2002.

4 World Health Organization: Definition and diagnosis of diabetes mellitus and intermediate hyperglycemia: a report of a WHO/IDF consultation. Geneva, World Health Organization, 2006, pp 1-46.

5 American Diabetes Association: Standards of medical care in diabetes - 2014. Diabetes Care 2014;37(suppl 1):S14-S80.

6 Diagnostic criteria and classification of hyperglycaemia first detected in pregnancy: a World Health Organization Guideline. Diabetes Res Clin Pract 2014;103:341-363.

7 Hughes RC, Moore MP, Gullam JE, Mohamed K, Rowan J: An early pregnancy HbAlc $>5.9 \%$ is optimal for detecting diabetes and identifies women at increased risk of adverse pregnancy outcomes. Diabetes Care 2014;37:2953-2959.

8 Fong A, Serra AE, Gabby L, Wing DA, Berkowitz KM: Use of hemoglobin A1c as an early predictor of gestational diabetes mellitus. Am J Obstet Gynecol 2014;211:641.e1641.e7.

9 Andraweera PH, Dekker GA, Roberts CT: The vascular endothelial growth factor family in adverse pregnancy outcomes. Hum Reprod Update 2012;18:436-457.

10 Redman CW, Sargent IL: Placental stress and pre-eclampsia: a revised view. Placenta 2009; 30(suppl A):S38-S42.

11 Akolekar R, Zaragoza E, Poon LC, Pepes S, Nicolaides KH: Maternal serum placental growth factor at $11+0$ to $13+6$ weeks of gestation in the prediction of pre-eclampsia. Ultrasound Obstet Gynecol 2008;32:732-739.

12 Poon LC, Zaragoza E, Akolekar R, Anagnostopoulos E, Nicolaides KH: Maternal serum placental growth factor (PlGF) in small for gestational age pregnancy at $11(+0)$ to $13(+6)$ weeks of gestation. Prenat Diagn 2008;28: $1110-1115$.

13 Ong CY, Lao TT, Spencer K, Nicolaides KH: Maternal serum level of placental growth factor in diabetic pregnancies. J Reprod Med 2004;49:477-480.

14 Tsiakkas A, Duvdevani N, Wright A, Wright D, Nicolaides KH: Serum placental growth factor in the three trimesters of pregnancy: effects of maternal characteristics and medical history. Ultrasound Obstet Gynecol 2015;45: 591-598.

15 Eleftheriades M, Papastefanou I, Lambrinoudaki I, Kappou D, Lavranos D, Akalestos A, Souka AP, Pervanidou P, Hassiakos D, Chrousos GP: Elevated placental growth factor concentrations at 11-14 weeks of gestation to predict gestational diabetes mellitus. Metabolism 2014;63:1419-1425.

16 Weycamp CW, Mosca A, Gillery P, Panteghini M: The analytical goals for hemoglobin $\mathrm{A}(1 \mathrm{c})$ measurement in IFCC units and national glycohemoglobin standardization program units are different. Clin Chem 2011;57: 1204-1206.

17 Burtis CA, Ashwood ER, Bruns DE: Tietz Textbook of Clinical Chemistry and Molecular Diagnostics, ed 4. St. Louis, Elsevier Saunders, 2006.

18 Sacks DA, Hadden DR, Maresh M, Deerochanawong C, Dyer AR, Metzger BE, Lowe LP, Coustan DR, Hod M, Oats JJ, Persson B, Trimble ER; HAPO Study Cooperative Research Group: Frequency of gestational diabetes mellitus at collaborating centers based on IADPSG consensus panel-recommended criteria: the Hyperglycemia and Adverse Pregnancy Outcome (HAPO) Study. Diabetes Care 2012;35:526-528.

19 Cheng R, Ma JX: Angiogenesis in diabetes and obesity. Rev Endocr Metab Disord 2015;16: 67-75.

20 Geva E, Ginzinger DG, Zaloudek CJ, Moore DH, Byrne A, Jaffe RB: Human placental vascular development: vasculogenic and angiogenic (branching and nonbranching) transformation is regulated by vascular endothelial growth factor-A, angiopoietin-1, and angiopoietin-2. J Clin Endocrinol Metab 2002;87: 4213-4224.

21 Huynh J, Dawson D, Roberts D, Bentley-Lewis R: A systematic review of placental pathology and maternal diabetes mellitus. Placenta 2015;36:101-114.

22 Cvitic S, Desoye G, Hiden U: Glucose, insulin, and oxygen interplay in placental hypervascularisation in diabetes mellitus. Biomed Res Int 2014;2014:145846.

23 Lassance L, Miedl H, Absenger M, Diaz-Perez F, Lang U, Desoye G, Hiden U: Hyperinsulinemia stimulates angiogenesis of human fetoplacental endothelial cells: a possible role of insulin in placental hypervascularization in diabetes mellitus. J Clin Endocrinol Metab 2013;98:e1438-e1447.

24 Loukovaara M, Leinonen P, Teramo K, Andersson S: Concentration of cord serum placenta growth factor in normal and diabetic pregnancies. BJOG 2005;112:75-79.

25 Gonzalez NL, Gonzalez DE, Castro A, Padron E, Plasencia W: Effect of pregestational diabetes mellitus on first trimester placental characteristics: three-dimensional placental volume and power Doppler indices. Placenta 2014;35:147-151.

26 Akolekar R, Syngelaki A, Poon LC, Wright D, Nicolaides KH: Competing risk model in early screening for preeclampsia by biophysical and biochemical markers. Fetal Diagn Ther 2013:33:8-15.

27 Cohen AL, Wenger JB, James-Todd T, Lamparello BM, Halprin E, Serdy S, Fan S, Horowitz GL, Kim KH, Rana S, Takoudes TC, Wyckoff JA, Thadhani R, Karumanchi SA, Brown FM: The association of circulating angiogenic factors and $\mathrm{HbAlc}$ with the risk of preeclampsia in women with preexisting diabetes. Hypertens Pregnancy 2014;33:8192.

28 International Expert Committee: International Expert Committee report on the role of the $\mathrm{A} 1 \mathrm{C}$ assay in the diagnosis of diabetes. Diabetes Care 2009;32:1327-1334.

29 Nicolaides KH: A model for a new pyramid of prenatal care based on the 11 to 13 weeks' assessment. Prenat Diagn 2011;31:3-6.

30 Syngelaki A, Pastides A, Kotecha R, Wright A, Akolekar R, Nicolaides KH: First-trimester screening for gestational diabetes mellitus based on maternal characteristics and history. Fetal Diagn Ther 2015;38:14-21.
First-Trimester PlGF in GDM and

Correlation of PlGF and HbAlc
Fetal Diagn Ther 5 